\title{
Training 100,000 Classes on a Single Titan X in 7 Hours or 15 Minutes with 25 Titan Xs ${ }^{*}$
}

\author{
Anshumali Shrivastava \\ Rice University \\ Houston, TX \\ anshumali@rice.edu
}

\begin{abstract}
In this talk, I will present Merged-Averaged Classifiers via Hashing (MACH) for $K$-classification with ultra-large values of $K$. Compared to traditional one-vs-all classifiers that require $O(K d)$ memory and inference cost, $\mathrm{MACH}$ only need $O(d \log K)(d$ is dimensionality) memory while only requiring $O(K \log K+d \log K)$ operation for inference. $\mathrm{MACH}$ is a generic $K$-classification algorithm, with provably theoretical guarantees, without any assumption on the relationship between classes. $\mathrm{MACH}$ uses universal hashing to reduce classification with a large number of classes to few (logarithmic many) independent classification tasks with small (constant) number of classes. I will show the first quantification of discriminabilitymemory tradeoff in multi-class classification. Using the simple idea of hashing, we can train ODP dataset with 100,000 classes and 400,000 features on a single Titan X GPU, with the classification accuracy of $19.28 \%$, which is the best-reported accuracy on this dataset. Before this work, the best performing baseline is a onevs-all classifier that requires 40 billion parameters (160 GB model size) and achieves $9 \%$ accuracy. In contrast, $\mathrm{MACH}$ can achieve $9 \%$ accuracy with $480 \mathrm{x}$ reduction in the model size (of mere $0.3 \mathrm{~GB}$ ). With $\mathrm{MACH}$, we also demonstrate complete training of feature extracted fine-grained imagenet dataset (compressed size 104GB), with 21,000 classes, on a single GPU. To the best of our knowledge, this is the first work to demonstrate complete training of these extreme-class datasets on a single Titan X. Furthermore, the algorithm is trivially parallelizable. Our experiments show that we can train ODP datasets in 7 hours on a single GPU or in 15 minutes with 25 GPUs. Similarly, we can train classifiers over the fine-grained imagenet dataset in 24 hours on a single GPU which can be reduced to little over 1 hour with 20 GPUs.
\end{abstract}

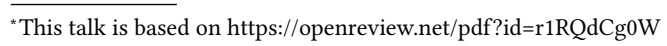

This paper is published under the Creative Commons Attribution 4.0 International (CC BY 4.0) license. Authors reserve their rights to disseminate the work on their personal and corporate Web sites with the appropriate attribution.

WWW'18 Companion, April 23-27, 2018, Lyon, France

() 2018 IW3C2 (International World Wide Web Conference Committee), published

under Creative Commons CC BY 4.0 License.

ACM ISBN 978-1-4503-5640-4/18/04.

https://doi.org/10.1145/3184558.3193135

\section{CCS CONCEPTS}

- Mathematics of computing $\rightarrow$ Probabilistic algorithms; Information theory; • Information systems $\rightarrow$ Clustering and classification; • Theory of computation $\rightarrow$ Distributed algorithms; • Computing methodologies $\rightarrow$ Parallel computing methodologies; Machine learning; Supervised learning by classification; Neural networks;

\section{KEYWORDS}

Large-scale Learning, Extreme Classification, Hashing, Sketching, Distributed Machine Learning, GPU

\section{ACM Reference Format:}

Anshumali Shrivastava. 2018. Training 100,000 Classes on a Single Titan X in 7 Hours or 15 Minutes with 25 Titan Xs. In WWW'18 Companion: The 2018 Web Conference Companion, April 23-27, 2018, Lyon, France. ACM, New York, NY, USA, 1 page. https://doi.org/10.1145/3184558.3193135 